\title{
A Uniformly Convergent Collocation Method for Singularly Perturbed Delay Parabolic Reaction-Diffusion Problem
}

\author{
Fasika Wondimu Gelu $\mathbb{D D}^{1}$ and Gemechis File Duressa $\mathbb{D}^{2}$ \\ ${ }^{1}$ Department of Mathematics, Dilla University, 419 Dilla, Ethiopia \\ ${ }^{2}$ Department of Mathematics, Jimma University, 378 Jimma, Ethiopia \\ Correspondence should be addressed to Gemechis File Duressa; gammeef@gmail.com
}

Received 6 September 2020; Accepted 22 February 2021; Published 13 March 2021

Academic Editor: Victor Kovtunenko

Copyright ( 2021 Fasika Wondimu Gelu and Gemechis File Duressa. This is an open access article distributed under the Creative Commons Attribution License, which permits unrestricted use, distribution, and reproduction in any medium, provided the original work is properly cited.

In this article, a numerical solution is proposed for singularly perturbed delay parabolic reaction-diffusion problem with mixedtype boundary conditions. The problem is discretized by the implicit Euler method on uniform mesh in time and extended cubic B-spline collocation method on a Shishkin mesh in space. The parameter-uniform convergence of the method is given, and it is shown to be $\varepsilon$-uniformly convergent of $O\left(\Delta t+N^{-2} \ln ^{2} N\right)$, where $\Delta t$ and $N$ denote the step size in time and number of mesh intervals in space, respectively. The proposed method gives accurate results by choosing suitable value of the free parameter $\lambda$. Some numerical results are carried out to support the theory.

\section{Introduction}

Let $\Omega=(0,1), D=\Omega \times(0, T]$, and $\Gamma=\Gamma_{l} \cup \Gamma_{b} \cup \Gamma_{r}$, where $\Gamma_{l}$ and $\Gamma_{r}$ are the left and right sides of the rectangular domain $D$ corresponding to $x=0$ and $x=1$, respectively, and $\Gamma_{b}$ is the base of the domain and given by $\Gamma_{b}=[0,1] \times[-\tau, 0]$. Note that $\Gamma_{l}=\{0\} \times(0, T]$ and $\Gamma_{r}=\{1\} \times(0, T]$. In this work, we consider the following singularly perturbed delay parabolic reaction-diffusion problem

$L u \equiv u_{t}-\varepsilon u_{x x}+a(x, t) u(x, t)=-b(x, t) u(x, \mathrm{t}-\tau)+f(x, t), \quad(x, t) \in D$,

subject to the initial condition

$$
u(x, t)=\phi_{b}(x, t), \quad(x, t) \in \Gamma_{b},
$$

and boundary conditions

$$
\begin{cases}B_{l} u(x, t) \equiv u(x, t)-\sqrt{\varepsilon} u_{x}(x, t)=\phi_{l}(t), & (x, t) \in \Gamma_{l}, \\ B_{r} u(x, t) \equiv u(x, t)+\sqrt{\varepsilon} u_{x}(x, t)=\phi_{r}(t), & (x, t) \in \Gamma_{r},\end{cases}
$$

where $\varepsilon(0<\varepsilon<<1)$ is a diffusion parameter whose presence makes the problem singularly perturbed and $\tau>0$ is a delay parameter. For the uniqueness of the solution, we assume that the functions involved in problem (1)-(3) are sufficiently smooth and bounded functions satisfying the following conditions

$$
\begin{gathered}
a(x, t) \geq \alpha>0, \\
b(x, t) \geq \beta>0, \\
(x, t) \in \bar{D} .
\end{gathered}
$$

This type of problem arises in various areas of science and engineering. The problem with delay occurs in the field of many biological models like epidemiology and population ecology. A typical example of delay parabolic partial differential equation is the temporal Wazewska-Czyzewska and Lasota equation which describes the survival of red blood cells in animals. This equation may be extended by incorporating a spatial component. The spatiotemporal delay reaction-diffusion equation of the following form is discussed in $[1,2]$ 


$$
\frac{\partial p(x, t)}{\partial t}=d \frac{\partial^{2} p(x, t)}{\partial x^{2}}-\delta p(x, t)+q e^{-a p(x, t-\tau)}
$$

where $\Omega \subset \mathbb{R}$ is a bounded domain and $(x, t) \in \Omega \times(0, \infty)$. The state variable $p(x, t)$ denotes the number of red blood cells located at $x$ at time $t>0$. The constant time delay $\tau$ denotes the time needed to produce blood cells. The parameter $\delta$ is the death rate of red blood cells, where the parameters $q$ and $a$ are related to the generation of red blood cells.

The numerical solution of delay partial differential equation depends not only on the solution at a present stage but also at some past stages. Many researchers have proposed different numerical methods to solve singularly perturbed time delay parabolic reaction-diffusion equations; for instance, see [3-8]. Singularly perturbed two-parameter time delay parabolic problems are studied in [9] using hybrid method based on a uniform mesh in time and a layer-adapted Shishkin mesh in space. In [10], the authors studied singularly perturbed differential-difference convection-diffusion equations using a higher order numerical method. But all the mentioned authors considered singularly perturbed time delay parabolic reaction-diffusion equations subject to Dirichlet boundary conditions. The authors in [11] studied singularly perturbed time delay parabolic reaction-diffusion equation subject to mixed-type boundary conditions, yet to the best of our knowledge, no further study has been done. In this work, we apply the extended form of cubic B-spline collocation method for singularly perturbed time delay parabolic reaction-diffusion problem subject to mixed boundary conditions.

B-spline functions have emerged as powerful techniques in the numerical solution of linear and nonlinear partial differential equations. B-spline functions are piecewise polynomial or nonpolynomial functions. B-spline functions were first formulated in 1946. Extended cubic B-spline is an improved version of cubic B-spline, where its basis is constructed in such a way that one free parameter, $\lambda$, is included and the degree of the piecewise polynomial is increased but the continuity of the extended cubic B-splines remains in order three. In [12], the authors proposed an extension of cubic B-spline of degree four with one free parameter, $\lambda$, which is called a shape parameter. This parameter is introduced within the basis function so as to change the shape of the spline. The authors in [13] generalized the extension to degrees five and six. Different researchers used extended cubic B-spline basis function to solve linear and nonlinear ordinary and partial differential equations; for example, see [14-16]. The authors in $[17,18]$ studied singularly perturbed semilinear differential equation of reaction-diffusion and convection-diffusion type, respectively, using cubic B-spline collocation method on a piecewise Shishkin mesh. Recently, cubic B-spline collocation method has been developed for time-dependent singularly perturbed differential-difference equations; see $[19,20]$. An extended cubic B-spline collocation method has been developed for time-dependent singularly perturbed partial differential equations with time lag in [21] and singularly perturbed parabolic differentialdifference equation arising in computational neuroscience in [22]. Recently, scholars in [23-26] studied timedependent singularly perturbed parabolic partial differential equations.

This article is structured as follows. The properties of continuous problem are given in Section 2. In Section 3, the description of numerical scheme and bounds of error for time semidiscretization followed by spatial discretization using extended B-spline collocation method are discussed. Error analysis in spatial direction and the overall error bounds are given in Section 4. Some numerical computations are given in Section 5. The conclusion is given in Section 6.

\section{Properties of Continuous Problem}

In the study of the numerical aspects of singularly perturbed problems, their analytical aspects play an important role. Setting the value $\varepsilon=0$, the reduced problem corresponding to (1)-(3) is

$$
\left\{\begin{array}{l}
\frac{\partial u_{0}(x, t)}{\partial t}+a(x, t) u_{0}(x, t)=-b(x, t) u_{0}(x, t-\tau)+f(x, t), \quad(x, t) \in D, \\
u(x, t)=\phi_{b}(x, t), \quad(x, t) \in \Gamma_{b} .
\end{array}\right.
$$

The reduced problem (6) is an initial value problem which will not make use of the two boundary conditions. As a result, the solution of problem in (1)-(3) will have left and right boundary layers. The characteristic curve of the reduced problem in equation (6) is the vertical lines $x=$ constant, which implies that boundary layers arising in the solution are of parabolic type. The problem in (1)-(3) satisfies the following continuous maximum principle.

Theorem 1. Assume that $a \in C^{(0,0)}(\bar{D})$ and let $\psi \in U^{*}=$ $C^{(2,1)}(D) \cap C^{(1,0)}\left(D^{*}\right) \cap C^{(0,0)}(\bar{D})$ be a sufficiently smooth function defined on $D$ such that $L \psi(x, t) \geq 0,(x, t) \in D, B_{l} \psi($ $x, t) \geq 0,(x, t) \in \Gamma_{l}, B_{r} \psi(x, t) \geq 0,(x, t) \in \Gamma_{r}$, and $\psi(x, t) \geq 0$, $(x, t) \in \Gamma_{0} \quad$ where $\quad D^{*}=D \cup \Gamma_{l} \cup \Gamma_{r}, \Gamma_{0}=(x, 0): x \in[0,1]$. Then, $\psi(x, t) \geq 0$, for all $(x, t) \in \bar{D}$.

Proof. The details of the proof are found in [11].

Stability and $\varepsilon$-uniform bound for problem (1) is established in the following theorem in the sense of the maximum norm which follows from Theorem 1.

Theorem 2. Let $v(x, t)$ be any function in the domain of problem. Then, we have the bound

$$
\|v\|_{\bar{D}} \leq \max \left\{\left\|B_{l} v(x, t)\right\|,\left\|B_{r} v(x, t)\right\|,\|v(x, t)\|, \frac{1}{\alpha}\|L v(x, t)\|\right\} .
$$

Proof. See the details of the proof in [11].

The existence and uniqueness for a solution of (1)-(3) can be established under the assumption that the data are Hölder continuous and also satisfy an appropriate 
compatibility conditions at the corner points $(0,0,1,0,0,-\tau)$ and $(1,-\tau)$. The boundary functions $\phi_{l}, \phi_{r} \in C^{k}([0, T]), \phi_{b} \in$ $C^{1, k}\left(\Gamma_{b}\right)$ are said to satisfy the $k^{\text {th }}$ order compatibility condition at the initial function if

$$
\begin{aligned}
& \frac{\partial^{k}}{\partial t^{k}}\left(\phi_{b}-\sqrt{\varepsilon} \frac{\partial \phi_{b}}{\partial x}\right)(0,0)=\frac{d^{k} \phi_{l}(0)}{\partial t^{k}}, \\
& \frac{\partial^{k}}{\partial t^{k}}\left(\phi_{b}+\sqrt{\varepsilon} \frac{\partial \phi_{b}}{\partial x}\right)(1,0)=\frac{d^{k} \phi_{r}(1)}{\partial t^{k}} .
\end{aligned}
$$

Therefore, the problem in (1)-(3) will have a unique solution which exhibits parabolic boundary layers at $x=0$ and $x=1$; see the details in $[3,11]$. Now, we establish the classical bounds on the solution and its derivatives.

Theorem 3. Let the coefficients and source function $a, b, f \in$ $C^{(2+\alpha, 1+\alpha / 2)}(\bar{D}), \phi_{l}, \phi_{r} \in C^{(3+\alpha) / 2}[(0, T)], \phi_{b} \in C^{(4+\alpha, 2+\alpha / 2)}\left(\Gamma_{b}\right), \alpha$ $\in(0,1)$. Assume that the compatibility conditions for $k=0$, 1,2 are fulfilled. Then, the problem has a unique solution and the derivatives of the solution $u$ satisfy the bound

$$
\left\|\frac{\partial^{i+j} u}{\partial x^{i} \partial t^{j}}\right\|_{\bar{D}} \leq C \varepsilon^{-\frac{i}{2}}, \quad i, j \geq 0,0 \leq i+2 j \leq 4,
$$

where the constant $C$ is independent of $\varepsilon$.

Proof. The proof of the first part is given in [27]. The bounds on the solution and its derivatives are explained in [11].

The classical bounds in Theorem 3 are not adequate for the proof of $\varepsilon$-uniform error estimate. Thus, the nonclassical bounds in singular and regular components and its derivatives are established in the following theorem.

Theorem 4. Let the coefficients and source function $a, b, f \in$ $C^{(4+\alpha, 2+\alpha / 2)}(\bar{D}), \phi_{l}, \phi_{r} \in C^{(5+\alpha) / 2}([0, T]), \phi_{b} \in C^{(6+\alpha, 3+\alpha / 2)}\left(\Gamma_{b}\right), \alpha$ $\in(0,1)$. Under the smoothness and compatibility conditions, we have the bounds for $i, j \geq 0,0 \leq i+2 j \leq 4$

$$
\begin{gathered}
\left\|\frac{\partial^{i+j} v(x, t)}{\partial x^{i} \partial t^{j}}\right\| \leq C\left(1+\varepsilon^{1-i / 2}\right), \\
\left\|\frac{\partial^{i+j} w_{l}}{\partial x^{i} \partial t^{j}}\right\| \leq C \varepsilon^{-\frac{i}{2}} \exp \left(-\frac{x}{\sqrt{\varepsilon}}\right), \\
\left\|\frac{\partial^{i+j} w_{r}}{\partial x^{i} \partial t^{j}}\right\| \leq C \varepsilon^{-\frac{i}{2}} \exp \left(-\frac{(1-x)}{\sqrt{\varepsilon}}\right) .
\end{gathered}
$$

Proof. The details of the proof are found in $[11,27]$.

\section{Description of the Numerical Method}

In this section, we utilize the implicit Euler method to discretize time derivative and then we introduce a piecewise uniform Shishkin mesh to discretize the space derivative using the extended form of cubic B-spline collocation method for the linear differential equations resulted from the time semidiscretization.

3.1. Time Semidiscretization. We discretize time derivative in (1)-(3) by means of the implicit Euler scheme on a uniform mesh with step length of $\Delta t$ defined by

$$
\Omega_{t}^{M}=\left\{t_{j}: t_{j}=t_{0}+j \Delta t, j=1, \cdots, M, t_{0}=0, \Delta t=\frac{T}{M}\right\},
$$

where $M$ denotes the number of mesh elements in temporal direction. Uniform meshes with step size $\Delta t, \Omega_{t}^{M}$ and $\Omega_{t}^{s}$ with $M$ and $s$ mesh elements are used on the interval $[0, T]$ and [ $-\tau, 0]$, respectively. The mesh size $\Delta t$ is chosen in such a way that the delay parameter $\tau=s \Delta t$, where $s$ is a positive integer, $t_{j}=j \Delta t, j \geq-s$. We obtain the following system of ordinary differential equations

$$
\left\{\begin{array}{l}
U^{j+1}(x)=\phi_{b}\left(x, t_{j+1}\right), \quad x \in \Omega,-(s+1) \leq j \leq 0, \\
\frac{U^{j+1}-U^{j}}{\Delta t}-\varepsilon U_{x x}^{j+1}+(a U)^{j+1}=-b^{j+1} U^{j-s+1}+f^{j+1}
\end{array}\right.
$$

subject to the boundary conditions

$$
\begin{cases}U^{j+1}(0)-\sqrt{\varepsilon} U_{x}^{j+1}(0)=\phi_{l}\left(t_{j+1}\right), & 0 \leq j \leq M, \\ U^{j+1}(1)+\sqrt{\varepsilon} U_{x}^{j+1}(1)=\phi_{r}\left(t_{j+1}\right), & 0 \leq j \leq M,\end{cases}
$$

where $U^{j+1}(x)=U\left(x, t_{j+1}\right)$ and $U^{j+1}$ is the numerical solution at the $(j+1)$ th time level. For each time step, equations (12)-(13) can be rewritten as

$$
-\varepsilon U_{x x}^{j+1}+(p U)^{j+1}=R^{j+1}
$$

subject to the initial and boundary conditions, respectively,

$$
U^{j+1}(x)=\phi_{b}\left(x, t_{j+1}\right), \quad x \in \Omega,-(s+1) \leq j \leq 0,
$$

$$
\begin{cases}U^{j+1}(0)-\sqrt{\varepsilon} U_{x}^{j+1}(0)=\phi_{l}\left(t_{j+1}\right), & 0 \leq j \leq M, \\ U^{j+1}(1)+\sqrt{\varepsilon} U_{x}^{j+1}(1)=\phi_{r}\left(t_{j+1}\right), & 0 \leq j \leq M .\end{cases}
$$

Here, $p^{j+1}=a^{j+1}+(1 / \Delta t), p^{j+1} \geq \alpha>0$ and $R^{j+1}=\left(U^{j} / \Delta t\right.$ )$-b^{j+1} U^{j-s+1}+f^{j+1}$. 
By using the initial condition, we can evaluate the righthand side as

$$
R^{j+1}(x)= \begin{cases}\frac{U^{j}}{\Delta t}-b^{j+1} \phi_{b}\left(x, t_{j-s+1}\right)+f^{j+1}, & j=0,1, \cdots, s, \\ \frac{U^{j}}{\Delta t}-b^{j+1} U\left(\mathrm{x}, t_{j-s+1}\right)+f^{j+1}, & j=s+1, \cdots, M .\end{cases}
$$

The local truncation error of an implicit Euler scheme for the temporal semidiscretization is given by $e_{j+1}=u\left(x, t_{j+1}\right)$ $-U^{j+1}(x)$. This error measures the contribution of each time step to the global error of the time semidiscretization.

Lemma 5. If

$$
\left|\frac{\partial^{i} u(x, t)}{\partial x^{i}}\right| \leq C, \quad(x, t) \in \bar{D}, 0 \leq i \leq 2,
$$

then the local error bound in the temporal direction is given by

$$
\left\|e_{j+1}\right\|_{\infty} \leq C(\Delta t)^{2}
$$

Proof. For the proof of the lemma, the readers can refer to [28].

The global error is the measure of the contribution of the local error estimate at each time step and is given by $e_{j}=u($ $\left.x, t_{j}\right)-U^{j}(x)$.

Lemma 6. Under the hypothesis of Lemma5, the global error estimate at $t_{j}$ is given by

$$
\left\|E_{j}\right\|_{\infty} \leq C \Delta t, j \leq \frac{T}{\Delta t}
$$

We conclude that time semidiscretization is first-order uniformly convergent.

3.2. Spatial Discretization. We first construct nonequidistant (layer adapted) Shishkin mesh as follows. We divide the three nonoverlapping subintervals $[0, \sigma],(\sigma, 1-\sigma)$, and $[1-\sigma, 1]$ into $N / 4, N / 2$, and $N / 4$ equidistant subintervals. We define the transition parameter $\sigma$ as $\sigma=\min \{1 / 4,2 \sqrt{\varepsilon} \ln (N)\}$. Let $\bar{\Omega}_{x}^{N}=\left\{x_{i}\right\}_{i=0}^{N}$ be the set of mesh points. Now, we define piecewise uniform mesh points as

$$
x_{i}= \begin{cases}i h_{i}, & \text { if } i=0(1) \frac{N}{4} \\ \sigma+\left(i-\frac{N}{4}\right) h_{i}, & \text { if } i=\left(\frac{N}{4}+1\right)(1) \frac{3 N}{4} \\ 1-\sigma+\left(i-\frac{3 N}{4}\right) h_{i}, & \text { if } i=\left(\frac{3 N}{4}+1\right)(1) N\end{cases}
$$

with piecewise uniform mesh spacing $h_{i}=4 \sigma / N$, if $i=1(1)($ $N / 4), i=((3 N / 4)+1)(1) N$, and $h_{i}=(2(1-2 \sigma)) / N$, if $i=(($ $N / 4)+1,1)(3 N / 4)$. Now, we apply the extended cubic Bspline collocation method to find the approximate solution for problem (14)-(16). Let $\Delta: 0=x_{0}<\cdots<x_{N}=1$ be the spatial domain $[0,1]$ with a piecewise uniform mesh spacing $h_{i}=x_{i+1}-x_{i}$. The extended form of cubic B-spline of degree $4, E_{i}(x, \lambda), i=-1,0,1, \cdots, N+1$, is defined by $[21,29]$

$$
E_{i}(x, \lambda)=\frac{1}{24 h_{i}^{4}} \begin{cases}4 h_{i}(1-\lambda)\left(x-x_{i-2}\right)^{3}+3 \lambda\left(x-x_{i-2}\right)^{4}, & x \in I_{i}, \\ (4-\lambda) h_{i}^{4}+12 h_{i}^{3}\left(x-x_{i-1}\right) & \\ +6 h_{i}^{2}(2+\lambda)\left(x-x_{i-1}\right)^{2} & \\ -12 h_{i}\left(x-x_{i-1}\right)^{3}-3 \lambda\left(x-x_{i-1}\right)^{4}, & x \in I_{i+1}, \\ (4-\lambda) h_{i}^{4}+12 h_{i}^{3}\left(x_{i+1}-x\right) & \\ +6 h_{i}^{2}(2+\lambda)\left(x_{i+1-x}\right)^{2} & \\ -12 h_{i}\left(x_{i+1}-x\right)^{3}-3 \lambda\left(x_{i+1}-x\right)^{4}, & x \in I_{i+2}, \\ 4 h_{i}(1-\lambda)\left(x_{i+2}-x\right)^{3}+3 \lambda\left(x_{i+2}-x\right)^{4}, & x \in I_{i+3}, \\ 0, & \text { otherwise, }\end{cases}
$$

where $I_{i}=\left[x_{i-2}, x_{i-1}\right]$ and $-m(m-2) \leq \lambda \leq 1$ is a free parameter which is used to change the shape of the B-spline curve and $m$ is the degree of extended cubic B-spline. The variation in $m$ gives different forms of extended cubic B-spline functions [29]. The extended cubic B-spline function has one free parameter $\lambda$, when the free parameter $\lambda$ tends to zero the extended cubic B-spline reduced to convectional cubic Bspline functions. For $\lambda \in[-8,1]$, cubic B-spline and extended cubic B-spline share the same properties such as local support, nonnegativity, partition of unity, and $C^{2}$ continuity; the parameter $\lambda$ controls the tension of the solution curve. The shape of extended cubic B-spline functions forces us to add two fictitious points $E_{-1}$ and $E_{N+1}$ to satisfy the boundary conditions. Since B-splines of degree $p$ are $(p-1)$ times continuously differentiable piecewise polynomials that form a basis of the space of splines, let $\varphi_{3}(\Omega)$ be the space of twice continuously differentiable piecewise extended cubic Bspline on $\Omega$. Since each $E_{i}(x)$ is also a piecewise cubic with knots at $\Omega$, each $E_{i}(x) \in \varphi_{3}(\Omega)$. Suppose that $E_{3}(\Omega)=$ span $\left\{E_{-1}, E_{0}, \cdots, E_{N}, E_{N+1}\right\}$. Since the functions $E_{i}{ }^{\prime}$ s are linearly independent on $[0,1], E_{3}(\Omega)$ is an $(N+3)$-dimensional. Let $S(x, \lambda)$ be the B-spline interpolating function for $u(x, t)$ at the nodal points and $S(x, \lambda) \in E_{3}(\Omega)$. Therefore, we seek an approximate solution $S(x, \lambda)$ of the problem (14)-(16) which is given by

$$
S(x, \lambda)=\sum_{i=-1}^{N+1} \gamma_{i} E_{i}(x, \lambda),
$$

where $\gamma_{i}$ are unknown real coefficients to be determined by requiring that $S(x, \lambda)$ satisfies (14)-(16) at $N+1$ collocation points and boundary conditions. The values of extended Bsplines $E_{i}(x, \lambda)$ and its derivatives at the nodal points can be calculated from (23) and depicted in Table 1. 
TABLe 1: Values of $E_{i}(x, \lambda)$ and its derivatives at nodal points.

\begin{tabular}{cccccc}
\hline & $x_{i-2}$ & $x_{i-1}$ & $x_{i}$ & $x_{i+1}$ & $x_{i+2}$ \\
\hline$E_{i}(x, \lambda)$ & 0 & $\frac{4-\lambda}{24}$ & $\frac{8+\lambda}{12}$ & $\frac{4-\lambda}{24}$ & 0 \\
$E_{i}^{\prime}(x, \lambda)$ & 0 & $\frac{1}{2 h_{i}}$ & 0 & $-\frac{1}{2 h_{i}}$ & 0 \\
$E_{i}^{\prime}(x, \lambda)$ & 0 & $\frac{2+\lambda}{2 h_{i}^{2}}$ & $-\frac{2+\lambda}{h_{i}^{2}}$ & $\frac{2+\lambda}{2 h_{i}^{2}}$ & 0 \\
\hline
\end{tabular}

An approximate solution over typical subinterval $\left[x_{i}\right.$, $\left.x_{i+1}\right]$ can be defined as

$$
S(x, \lambda)=\sum_{j=i-1}^{i+2} \gamma_{j} E_{j}(x, \lambda) .
$$

Now, substituting the values of $E_{i}$ for $S_{i}(\lambda)$ and $E_{i}{ }^{\prime \prime}$ for $S_{i}{ }^{\prime \prime}(\lambda)$ as stated in Table 1 in equation (14), we get $(N+1)$ linear equations in $(N+3)$ unknowns as

$$
r_{i}^{-} \gamma_{i-1}+r_{i}^{c} \gamma_{i}+r_{i}^{+} \gamma_{i+1}=\tilde{R}_{i}, \quad \text { for } i=0,1, \cdots, N \text {, }
$$

where the coefficients are given by

$$
\left\{\begin{array}{l}
r_{i}^{-}=\frac{-\varepsilon(2+\lambda)}{2 h_{i}^{2}}+\frac{(4-\lambda)}{24} \tilde{p}_{i} \\
r_{i}^{c}=\frac{\varepsilon(2+\lambda)}{h_{i}^{2}}+\frac{(8+\lambda)}{12} \tilde{p}_{i} \\
r_{i}^{+}=\frac{-\varepsilon(2+\lambda)}{2 h_{i}^{2}}+\frac{(4-\lambda)}{24} \tilde{p}_{i} \\
\tilde{p}_{i}=p_{i}^{j+1}, \tilde{R}_{i}=R_{i}^{j+1}
\end{array}\right.
$$

Boundary conditions in (16) at $x_{0}$ and $x_{N}$ must be imposed to the system of equations in (25) to obtain the unique solution. Thus, we obtain the approximate solution at two boundary points as follows:

$$
\begin{gathered}
a_{1} \gamma_{-1}+b_{1} \gamma_{0}+c_{1} \gamma_{1}=\phi_{l}\left(t_{j+1}\right), \\
c_{1} \gamma_{N-1}+b_{1} \gamma_{N}+a_{1} \gamma_{N+1}=\phi_{r}\left(t_{j+1}\right)
\end{gathered}
$$

where the coefficients are given by $a_{1}=((4-\lambda) / 24)-(\sqrt{\varepsilon} /$ $\left.2 h_{i}\right), b_{1}=(8+\lambda) / 12, c_{1}=((4-\lambda) / 24)+\left(\sqrt{\varepsilon} / 2 h_{i}\right)$. Equations (25) and (27) lead to $(N+3) \times(N+3)$ linear systems with $(N+3)$ unknowns $\gamma_{-1}, \gamma_{0}, \cdots, \gamma_{N+1}$. Excluding the unknowns $\gamma_{-1}$ and $\gamma_{N+1}$ from (27) for $i=0$ and $i=N$, then (25) becomes solvable system of $(N+1) \times(N+1)$ linear equations in $(N$ +1 ) unknowns $\gamma_{0}, \cdots, \gamma_{N}$ in matrix form as

$$
M \gamma=G, \quad i=1,2, \cdots, N-1
$$

where the entries of the tridiagonal matrix $M=\left(m_{i j}\right)$ are given by

$$
m_{i j}= \begin{cases}r_{0}^{c}-\frac{b_{1}}{a_{1}} r_{0}^{-}, & i=j=0, \\ r_{0}^{+}-\frac{c_{1}}{a_{1}} r_{0}^{-}, & i=0, j=1 \\ r_{i}^{-}, & i=j+1, j=0(1) N-2, \\ r_{i}^{c}, & i=j=1(1) N-1, \\ r_{i}^{+}, & i=j-1, j=2(1) N, \\ r_{N}^{-}-\frac{c_{1}}{a_{1}} r_{N}^{+}, & i=N, j=N-1, \\ r_{N}^{c}-\frac{b_{1}}{a_{1}} r_{N}^{+}, & i=j=N, \\ 0, & \forall|i-j|>0 .\end{cases}
$$

The entries of the column vector $G$ are given as

$$
G= \begin{cases}\tilde{R}_{0}^{j+1}-\frac{r_{0}^{-}}{a_{1}} \phi_{l}\left(t_{j+1}\right), & j=0, \\ \tilde{R}_{i}^{j+1}, & j=1(1) N-1, \\ \tilde{R}_{N}^{j+1}-\frac{r_{N}^{+}}{a_{1}} \phi_{r}\left(t_{j+1}\right), & j=N .\end{cases}
$$

Since $p_{i}^{j+1}>0$, it is easily seen that for $\lambda>-2$ the matrix $M$ is strictly diagonally dominant and hence nonsingular. Since $M$ is nonsingular, we can solve the system (28) for $\gamma_{0}, \cdots, \gamma_{N}$. Hence, the extended cubic B-spline collocation method applied to problem (14) has a unique solution $S(x, \lambda)$.

\section{Error Analysis}

This section proves the $\varepsilon$-uniformly convergence of the proposed method in the spatial direction. For this, we use the following lemma.

Lemma 7. The extended cubic B-splines $\left\{E_{-1}(x, \lambda), E_{0}(x, \lambda)\right.$, $\left.\cdots, E_{N}(x, \lambda), E_{N+1}(x, \lambda)\right\}$ defined in (22) satisfy the following inequality

$$
\sum_{i=-1}^{N+1}\left|E_{i}(x, \lambda)\right| \leq \frac{7}{4}, \quad x \in[0,1] .
$$

Proof. We know that

$$
\left|\sum_{i=-1}^{N+1} E_{i}(x, \lambda)\right| \leq \sum_{i=-1}^{N+1}\left|E_{i}(x, \lambda)\right| .
$$

Extended cubic B-spline $E_{i}(x, \lambda)$ is nonzero at only three nodal points. Thus, at any nodal value $x_{i}$, from Table 1 we obtain 
$\sum_{i=-1}^{N+1}\left|E_{i}(x, \lambda)\right|=\left|E_{i-1}\left(x_{i}, \lambda\right)\right|+\left|E_{i}\left(x_{i}, \lambda\right)\right|+\left|E_{i+1}\left(x_{i}, \lambda\right)\right|=\frac{4-\lambda}{24}+\frac{8+\lambda}{12}+\frac{4-\lambda}{24}=1<\frac{7}{4}$

From Table 1 , for $x \in\left[x_{i-1}, x_{i}\right]$, we have

$$
\begin{gathered}
\left|E_{i}\left(x_{i}, \lambda\right)\right| \leq \frac{8+\lambda}{12}, \\
\left|E_{i-1}\left(x_{i-1}, \lambda\right)\right| \leq \frac{8+\lambda}{12} .
\end{gathered}
$$

Similarly, for $x \in\left[x_{i-1}, x_{i}\right]$, we have that

$$
\begin{gathered}
\left|E_{i+1}\left(x_{i}, \lambda\right)\right| \leq \frac{4-\lambda}{24}, \\
\left|E_{i-2}\left(x_{i-1}, \lambda\right)\right| \leq \frac{4-\lambda}{24} .
\end{gathered}
$$

Now, for any point for $x \in\left[x_{i-1}, x_{i}\right]$, we get

$\sum_{i=-1}^{N+1}\left|E_{i}(x, \lambda)\right|=\left|E_{i-1}(x, \lambda)\right|+\left|E_{i}(x, \lambda)\right|+\left|E_{i+1}(x, \lambda)\right|+\left|E_{i-2}(x, \lambda)\right|=\frac{20+\lambda}{12}$.

Since $-8<\lambda<1$, thus $(20+\lambda) / 12 \leq 7 / 4$ and this completes the proof.

Let $Y(x)$ be the unique cubic spline interpolate from an approximate solution $S(x, \lambda)$ of the problem (14)-(16) to the solution $u(x, t)$ which is given by

$$
Y(x)=\sum_{i=-1}^{N+1} \bar{\gamma}_{i} E_{i}(x, \lambda) .
$$

Lemma 8. Let $Y(x) \in C^{2}([0,1])$ be the cubic spline interpolant associated with a solution $\tilde{u}(x)$. If $\tilde{u}(x) \in C^{4}([0,1])$, it follows from the estimate of Hall [30] that the standard cubic spline interpolation error estimate holds, for $x \in \Omega_{i}:=\left[x_{i}, x_{i+1}\right] \in \Omega$

$$
\left\|D^{n}(\widehat{u}(x)-Y(x))\right\| \leq \lambda_{n}\left\|u \wedge^{(4)}(x)\right\| h_{i}^{4-n}, \quad n=0,1,2,3,
$$

where $\lambda_{n}$ are constants independent of $h_{i}$ and $N$.

Lemma 9. [31]. If matrix $A$ is strictly diagonally dominant by rows and constant $\zeta=\min _{i}\left(\left|a_{i, i}\right|-\sum_{i \neq j}\left|a_{i, j}\right|\right)$. Then, we have the bound

$$
\left\|A^{-1}\right\|_{\infty}<\frac{1}{\zeta}
$$

Theorem 10. Let $S(x, \lambda)$ be an extended cubic B-spline collocation approximation from the space of extended $B$-splines $\varphi_{3}(\Omega)$ to the solution of (14)-(16) and $\widehat{u}\left(x_{i}\right)$ is the analytical solution to the problem. If $\tilde{g}(x) \in C^{2}([0,1])$, then the parameter- uniform error estimate satisfies the bound

$$
\sup _{0<\varepsilon \leq 1} \max _{0 \leq i \leq N}\left\|\widehat{u}\left(x_{i}\right)-S\left(x_{i}, \lambda\right)\right\| \leq C N^{-2}(\ln N)^{2} \text {, }
$$

where $C$ is a constant independent of $\varepsilon$ and $N$.

Proof. To estimate the error $\left|\widehat{u}\left(x_{i}\right)-S\left(x_{i}, \lambda\right)\right|$, it follows immediately from the estimates in Lemma 8 and equation (37) that

$$
\begin{aligned}
\left|\widehat{L} \widehat{u}\left(x_{i}\right)-\widehat{L} Y\left(x_{i}\right)\right| & \leq \varepsilon\left(u \wedge^{\prime \prime}\left(x_{i}\right)-Y^{\prime \prime}\left(x_{i}\right)\right)+p\left(x_{i}\right)\left(\widehat{u}\left(x_{i}\right)-Y\left(x_{i}\right)\right) \mid \\
& \leq \varepsilon\left(\lambda_{2}\left|u \wedge^{(4)}\right| h_{i}^{2}\right)+\|p\|\left(\lambda_{0}\left|u \wedge^{(4)}\right| h_{i}^{4}\right),
\end{aligned}
$$

from (37).

Therefore, rearrangement of the above expression gives

$$
\left|\widehat{L} \widehat{u}\left(x_{i}\right)-\widehat{L} Y\left(x_{i}\right)\right| \leq\left(\varepsilon \lambda_{2} h_{i}^{2}+\|p\|_{\infty} \lambda_{0} h_{i}^{4}\right)\left|u \wedge^{(4)}\right| \text {. }
$$

Since the argument depends on whether $\sigma=1 / 4$ or $\sigma=$ $2 \sqrt{\varepsilon} \ln N<1 / 4$, there arises two cases.

Case 1. When $\sigma=1 / 4$, the mesh is uniform with spacing $1 / N$, that is, $h_{i}=1 / N$ and $2 \sqrt{\varepsilon} \ln N \geq 1 / 4$ gives $\varepsilon^{-1 / 2} \leq C \ln N$. From this, we get $\varepsilon^{-1} \leq(C \ln N)^{2}$. In this case, we use a classical analysis to prove convergence. Using the classical bound in Theorem 3, that is, $\left|u \wedge^{(4)}\right| \leq C \varepsilon^{-2}$ together with equation (42) yields

$$
\begin{aligned}
\left|\widehat{L} \widehat{u}\left(x_{i}\right)-\widehat{L} Y\left(x_{i}\right)\right| & \leq C \varepsilon^{-2}\left(\varepsilon \lambda_{2} N^{-2}+\|p\|_{\infty} \lambda_{0} N^{-4}\right) \\
& \leq C N^{-2}\left(\varepsilon^{-1}+\varepsilon^{-2} N^{-2}\right) \\
& \leq C N^{-2}\left((C \ln N)^{2}+C N^{-2}(\ln N)^{4}\right) .
\end{aligned}
$$

Since $C N^{-2}(\ln N)^{4} \leq C(\ln N)^{2}$, we obtain the following estimate:

$$
\left|\widehat{L} \widehat{u}\left(x_{i}\right)-\widehat{L} Y\left(x_{i}\right)\right| \leq C N^{-2}(\ln N)^{2} .
$$

Case 2. If $\Omega_{i}$ lies in the boundary layer regions, then mesh spacing $h_{i} \leq C \varepsilon^{1 / 2} N^{-1} \ln N$. Using the bound in the layer regions together with the estimates in equation (42), we have

$$
\begin{aligned}
\left|\widehat{L} \widehat{u}\left(x_{i}\right)-\widehat{L} Y\left(x_{i}\right)\right| & \leq C \varepsilon^{-2}\left(\varepsilon \lambda_{2} C^{2} \varepsilon N^{-2} \ln ^{2} N+\|p\|_{\infty} \lambda_{0} C^{4} \varepsilon^{2} N^{-4} \ln ^{4} N\right) \\
& \leq C N^{-2}\left((\ln N)^{2}+N^{-2}(\ln N)^{4}\right) .
\end{aligned}
$$
mate:

Since $N^{-2}(\ln N)^{4} \leq(\ln N)^{2}$, we obtain the following esti-

$$
\left|\widehat{L} \widehat{u}\left(x_{i}\right)-\widehat{L} Y\left(x_{i}\right)\right| \leq C N^{-2}(\ln N)^{2} .
$$


TABLE 2: The maximum point-wise errors $E_{\varepsilon}^{N, \Delta t}$ for various $\varepsilon$ and $\lambda=0.99$.

\begin{tabular}{lcccccc}
\hline$\varepsilon \downarrow$ & $N=32, \Delta t=0.1 / 2^{2}$ & $64,0.1 / 2^{3}$ & $128,0.1 / 2^{4}$ & $256,0.1 / 2^{5}$ & $512,0.1 / 2^{6}$ & $1024,0.1 / 2^{7}$ \\
\hline $2^{-6}$ & $9.7604 e-2$ & $4.9405 e-2$ & $2.9193 e-2$ & $1.7022 e-2$ & $4.4560 e-3$ & $2.1246 e-3$ \\
$2^{-7}$ & $9.7647 e-2$ & $4.9411 e-2$ & $2.4853 e-2$ & $1.4146 e-2$ & $4.4571 e-3$ & $2.1266 e-3$ \\
$2^{-8}$ & $9.7679 e-2$ & $4.9415 e-2$ & $2.4853 e-2$ & $1.2463 e-2$ & $5.1580 e-3$ & $2.1270 e-3$ \\
$2^{-9}$ & $1.1064 e-1$ & $4.9417 e-2$ & $2.4854 e-2$ & $1.2463 e-2$ & $5.1580 e-3$ & $2.1270 e-3$ \\
$2^{-10}$ & $1.1980 e-1$ & $5.5853 e-2$ & $2.4854 e-2$ & $1.2463 e-2$ & $5.1580 e-3$ & $2.1270 e-3$ \\
$2^{-11}$ & $1.1990 e-1$ & $5.6763 e-2$ & $2.6206 e-2$ & $1.2463 e-2$ & $5.1580 e-3$ & $2.1270 e-3$ \\
$2^{-12}$ & $1.1990 e-1$ & $5.6763 e-2$ & $2.6206 e-2$ & $1.2463 e-2$ & $5.1580 e-3$ & $2.1270 e-3$ \\
$2^{-13}$ & $1.1990 e-1$ & $5.6763 e-2$ & $2.6206 e-2$ & $1.2463 e-2$ & $5.1580 e-3$ & $2.1270 e-3$ \\
$2^{-14}$ & $1.1990 e-1$ & $5.6763 e-2$ & $2.6206 e-2$ & $1.2463 e-2$ & $5.1580 e-3$ & $2.1270 e-3$ \\
$2^{-15}$ & $1.1990 e-1$ & $5.6763 e-2$ & $2.6206 e-2$ & $1.2463 e-2$ & $5.1580 e-3$ & $2.1270 e-3$ \\
$2^{-16}$ & $1.1990 e-1$ & $5.6763 e-2$ & $2.6206 e-2$ & $1.2463 e-2$ & $5.1580 e-3$ & $2.1270 e-3$ \\
$\vdots$ & $\vdots$ & $\vdots$ & $\vdots$ & $\vdots$ & $\vdots$ \\
$2^{-28}$ & $1.1990 e-1$ & $5.6763 e-2$ & $2.6206 e-2$ & $1.2463 e-2$ & $5.1580 e-3$ & $2.1270 e-3$ \\
$2^{-29}$ & $1.1990 e-1$ & $5.6763 e-2$ & $2.6206 e-2$ & $1.2463 e-2$ & $5.1580 e-3$ & $2.1270 e-3$ \\
$2^{-30}$ & $1.1990 e-1$ & $5.6763 e-2$ & $2.6206 e-2$ & $1.2463 e-2$ & $5.1580 e-3$ & $2.1270 e-3$ \\
\hline
\end{tabular}

TABLE 3: Comparison of $\varepsilon$-uniform values for $\lambda=0.99$ using the proposed method and finite difference method.

\begin{tabular}{|c|c|c|c|c|c|}
\hline$\forall \varepsilon \downarrow$ & $N=32, \Delta t=0.1 / 2^{2}$ & $64,0.1 / 2^{3}$ & $128,0.1 / 2^{4}$ & $256,0.1 / 2^{5}$ & $512,0.1 / 2^{6}$ \\
\hline \multicolumn{6}{|c|}{ Our result } \\
\hline$E^{N, \Delta t}$ & $1.1990 e-1$ & $5.6763 e-2$ & $2.6206 e-2$ & $1.2463 e-2$ & $5.1580 e-3$ \\
\hline$R^{N, \Delta t}$ & 1.0788 & 1.1151 & 1.1722 & 1.2728 & 1.2780 \\
\hline \multicolumn{6}{|c|}{ Result in [11] } \\
\hline$E^{N, \Delta t}$ & $2.3070 e-1$ & $1.2002 e-1$ & $6.1238 e-2$ & $3.0931 e-2$ & $1.5545 e-2$ \\
\hline$R^{N, \Delta t}$ & 0.9427 & 0.9709 & 0.9853 & 0.9926 & 0.9963 \\
\hline
\end{tabular}

TABLE 4: Comparison of $\varepsilon$-uniform values for $\lambda=0$ and $\lambda=0.99$ using the proposed method.

\begin{tabular}{|c|c|c|c|c|c|}
\hline$\overline{\forall \varepsilon \downarrow}$ & $N=32, \Delta t=0.1 / 2^{2}$ & $64,0.1 / 2^{3}$ & $128,0.1 / 2^{4}$ & $256,0.1 / 2^{5}$ & $512,0.1 / 2^{6}$ \\
\hline \multicolumn{6}{|c|}{ For $\lambda=0$} \\
\hline$E^{N, \Delta t}$ & $1.5717 e-1$ & $7.3329 e-2$ & $3.3118 e-2$ & $1.4555 e-2$ & $6.9985 e-3$ \\
\hline$R^{N, \Delta t}$ & 1.0999 & 1.1468 & 1.1861 & 1.2232 & 1.2216 \\
\hline \multicolumn{6}{|c|}{ For $\lambda=0.99$} \\
\hline$E^{N, \Delta t}$ & $1.1990 e-1$ & $5.6763 e-2$ & $2.6206 e-2$ & $1.2463 e-2$ & $5.1580 e-3$ \\
\hline$R^{N, \Delta t}$ & 1.0788 & 1.1151 & 1.1722 & 1.2728 & 1.2780 \\
\hline
\end{tabular}

On the other hand, for the subinterval $[\sigma, 1-\sigma]$, that is, for the outer region, the mesh spacing is $h_{i}=2 N^{-1}(1-2 \sigma)$ $=2 N^{-1}-C \sqrt{\varepsilon} N^{-1} \ln N \leq C \varepsilon^{1 / 2} N^{-1} \ln N$. Using this in equation (42) together with the bounds gives us

$$
\begin{aligned}
\left|\widehat{L} \widehat{u}\left(x_{i}\right)-\widehat{L} Y\left(x_{i}\right)\right| & \leq C \varepsilon^{-2}\left(\varepsilon \lambda_{2} C^{2} \varepsilon N^{-2} \ln ^{2} N+\|p\|_{\infty} \lambda_{0} C^{4} \varepsilon^{2} N^{-4} \ln ^{4} \mathrm{~N}\right) \\
& \leq C N^{-2}\left((\ln N)^{2}+N^{-2}(\ln N)^{4}\right) .
\end{aligned}
$$

Since $N^{-2}(\ln N)^{4}$ is very small number, we obtain the following estimate:

$$
\left|\widehat{L} \widehat{u}\left(x_{i}\right)-\widehat{L} Y\left(x_{i}\right)\right| \leq C N^{-2}(\ln N)^{2} .
$$
have

Combining the above estimates for both the cases, we

$$
\left|\widehat{L} \widehat{u}\left(x_{i}\right)-\widehat{L} Y\left(x_{i}\right)\right| \leq C N^{-2}(\ln N)^{2} .
$$




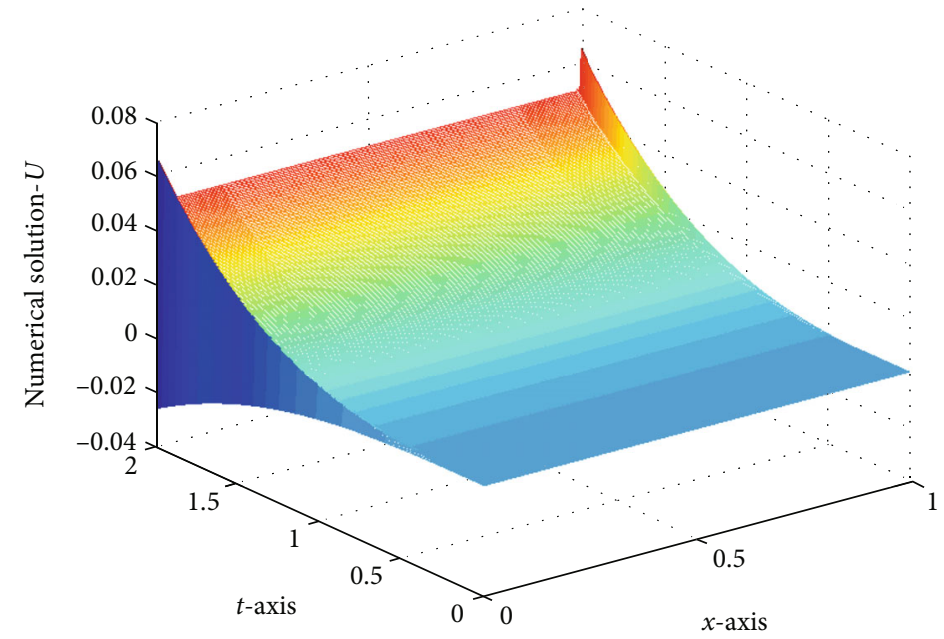

FIgURE 1: Numerical simulation at $N=2^{7}, \Delta t=0.1 / 2^{4}, \varepsilon=2^{-12}, \lambda=-0.55$.

Therefore, we have that

$\left|\widehat{L} S\left(x_{i}\right)-\widehat{L} Y\left(x_{i}\right)\right|=\left|\widehat{R}\left(x_{i}\right)-\widehat{L} Y\left(x_{i}\right)\right|=\left|\widehat{L} \widehat{u}\left(x_{i}\right)-\widehat{L} Y\left(x_{i}\right)\right| \leq C N^{-2}(\ln N)^{2}$.

We know that $\widehat{L} U\left(x_{i}\right)=\widehat{R}\left(x_{i}\right), 0 \leq i \leq N$ with the boundary conditions $U\left(x_{0}\right)-\sqrt{\varepsilon} U^{\prime}\left(x_{0}\right)=\phi_{l}\left(t_{j+1}\right)$ and $U\left(x_{N}\right)+$ $\sqrt{\varepsilon} U^{\prime}\left(x_{N}\right)=\phi_{r}\left(t_{j+1}\right)$ leads to the linear system $M \gamma=G$. Assume that $\widehat{L} Y\left(x_{i}\right)=\bar{R}\left(x_{i}\right), 0 \leq i \leq N$ with the boundary conditions $Y\left(x_{0}\right)-\sqrt{\varepsilon} Y^{\prime}\left(x_{0}\right)=\bar{\phi}_{l}\left(t_{j+1}\right)$ and $Y\left(x_{N}\right)+\sqrt{\varepsilon} Y^{\prime}($ $\left.x_{N}\right)=\bar{\phi}_{r}\left(t_{j+1}\right)$ leads to the linear system $M \bar{\gamma}=\bar{G}$. It follows that

$$
M(\gamma-\bar{\gamma})=(G-\bar{G})
$$

where $\gamma-\bar{\gamma}=\left(\gamma_{0}-\bar{\gamma}_{0}, \gamma_{1}-\bar{\gamma}_{1}, \cdots, \gamma_{N}-\bar{\gamma}_{N}\right)^{T}$ and

$$
G-\bar{G}=\left(\begin{array}{c}
\left(\tilde{R}_{0}-\bar{R}_{0}\right)+\frac{r_{0}^{-}}{a_{1}}\left(\bar{\phi}_{l}\left(t_{j+1}\right)-\phi_{l}\left(t_{j+1}\right)\right) \\
\left(\tilde{R}_{1}-\bar{R}_{1}\right) \\
\vdots \\
\left(\tilde{R}_{N-1}-\bar{R}_{N-1}\right) \\
\left(\tilde{R}_{N}-\bar{R}_{N}\right)+\frac{r_{N}^{+}}{a_{1}}\left(\bar{\phi}_{r}\left(t_{j+1}\right)-\phi_{r}\left(t_{j+1}\right)\right)
\end{array}\right) .
$$

Since $M \gamma-M \bar{\gamma}=G-\bar{G}$ implies that $\widehat{L} S\left(x_{i}\right)-\widehat{L} Y\left(x_{i}\right)$, from (50) we have

$$
\|G-\bar{G}\| \leq C N^{-2}(\ln N)^{2} .
$$

It can be seen that for reasonable large $N$, the matrix $M$ is strictly diagonally dominant and thus nonsingular. From the estimate in Lemma 9, we get

$$
\left\|M^{-1}\right\|_{\infty} \leq C
$$

Combining the bounds in equations (51)-(54), we obtain

$$
\|\gamma-\bar{\gamma}\| \leq C N^{-2}(\ln N)^{2}
$$

Let $e=\left(e_{0}, \cdots, e_{N}\right)^{T}$, where $e_{i}=\gamma_{i}-\bar{\gamma}_{i}$. Now, from (51), we have

$$
e=M^{-1}(G-\bar{G})
$$

Using (53) and (54) in (56), we have the following estimate:

$$
\|e\| \leq C N^{-2}(\ln N)^{2}
$$

We have

$$
\begin{gathered}
a_{1}\left(\gamma_{-1}-\bar{\gamma}_{-1}\right)+b_{1}\left(\gamma_{0}-\bar{\gamma}_{0}\right)+c_{1}\left(\gamma_{1}-\bar{\gamma}_{1}\right)=\phi_{l}\left(t_{j+1}\right), \\
c_{1}\left(\gamma_{N-1}-\bar{\gamma}_{N-1}\right)+b_{1}\left(\gamma_{N}-\bar{\gamma}_{N}\right)+a_{1}\left(\gamma_{N+1}-\bar{\gamma}_{N+1}\right)=\phi_{r}\left(t_{j+1}\right),
\end{gathered}
$$

where $a_{1}, b_{1}$, and $c_{1}$ are defined in (27). From this, it is a simple task to obtain $\left|\gamma_{-1}-\bar{\gamma}_{-1}\right| \leq C N^{-2}(\ln N)^{2}$ and $\mid \gamma_{N+1}-$ $\bar{\gamma}_{N+1} \mid \leq C N^{-2}(\ln N)^{2}$. Therefore, we have the following estimation from boundary conditions as

$$
\max _{-1 \leq i \leq N+1}\left|\gamma_{i}-\bar{\gamma}_{i}\right| \leq C N^{-2}(\ln N)^{2}
$$

Therefore, the above inequality enables us to estimate $\mid S$ $(x, \lambda)-Y(x) \mid$ as

$$
|S(x, \lambda)-Y(x)|=\sum_{i=-1}^{N+1}\left(\gamma_{i}-\bar{\gamma}_{i}\right)\left|E_{i}(x, \lambda)\right| .
$$




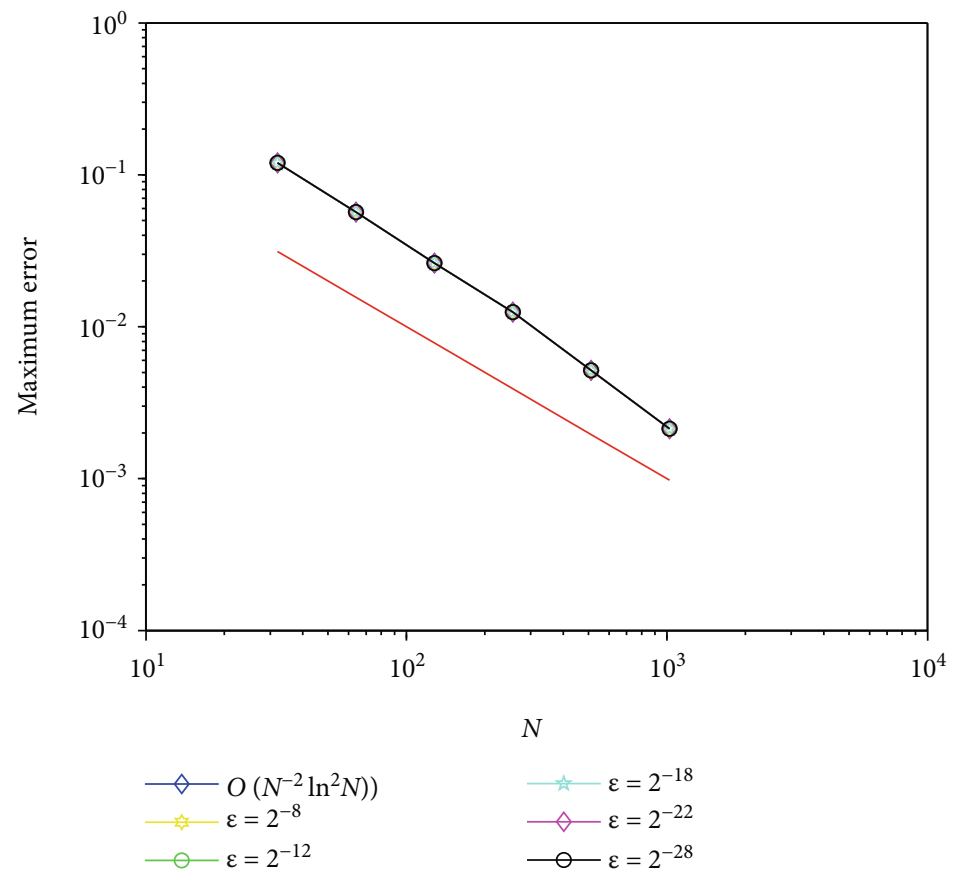

Figure 2: Plot of maximum-uniform errors in log-log scale.

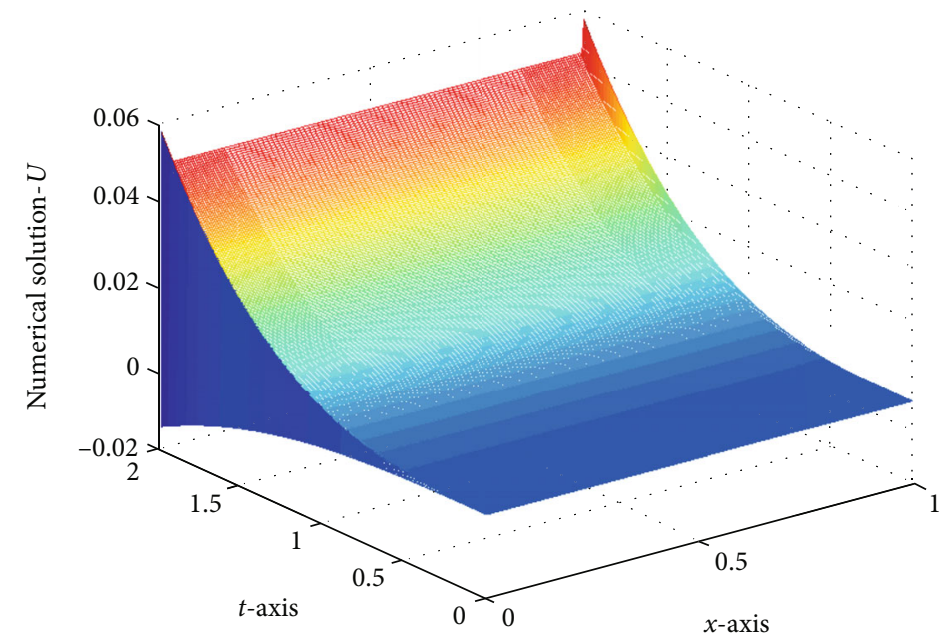

Figure 3: Numerical simulation at $N=2^{7}, \Delta t=0.1 / 2^{4}, \varepsilon=2^{-12}, \lambda=0$.

Using (56) and Lemma 7, we obtain

$$
\max _{0 \leq i \leq N}\left|S\left(x_{i}, \lambda\right)-Y\left(x_{i}\right)\right| \leq C N^{-2}(\ln N)^{2}
$$

Using triangle inequality and the results in (49) and (61) gives

$$
\sup _{0<\varepsilon \leq 1} \max _{0 \leq i \leq N}\left|\widehat{u}\left(x_{i}\right)-S\left(x_{i}, \lambda\right)\right| \leq C N^{-2}(\ln N)^{2} .
$$

Hence, this completes the proof.
Theorem 11. Let $S\left(x_{i}, \lambda\right)$ be the extended B-spline collocation approximation to the solution $u(x, t)$ of the problem (14)-(16). Then, the parameter-uniform error estimate of fully discrete scheme is given by

$$
\left|u\left(x_{i}\right)-S\left(x_{i}, \lambda\right)\right| \leq C\left(\Delta t+N^{-2} \ln ^{2} N\right)
$$

where $C$ is a constant independent of mesh parameters and $\varepsilon$.

Proof. The result of Lemma 6 and Theorem 10 proves this theorem. 


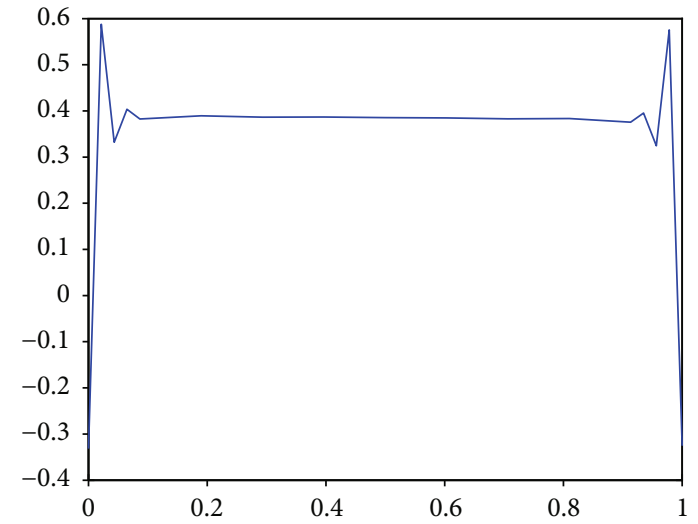

FIgURE 4: Line graph at $N=2^{4}, \Delta t=0.1 / 2, \varepsilon=2^{-12}, \lambda=-0.55$.

\section{Numerical Results and Discussions}

To see the applicability and efficiency of the proposed method, an example is considered from the literature. Computations are done for reasonable value of the free parameter $\lambda=0.99 \in[-8,1]$, which gives minimum error. Since the exact solution for the test example is unknown, we use the double mesh principle to calculate absolute errors. For each $\mathcal{E}$, we can determine the maximum point-wise errors using the formula as

$$
E_{\varepsilon}^{N, \Delta t}=\max _{0 \leq i \leq N ; t \in[0, T]}\left|U^{N, \Delta t}\left(x_{i}, t_{j}\right)-U^{2 N, 2 M}\left(x_{2 i}, t_{2 j}\right)\right|,
$$

where $U^{N, \Delta t}\left(x_{i}, t_{j}\right)$ denote the numerical solution obtained at $(N, \Delta t)$ mesh points whereas $U^{2 N, \Delta t / 2}\left(x_{2 i}, t_{2 j}\right)$ denote the numerical solution at $(2 N, 2 M)$ mesh points. The uniform rate of convergence is calculated by the formula

$$
R^{N, \Delta t}=\log _{2}\left(\frac{E^{N, \Delta t}}{E^{2 N . \Delta t / 2}}\right) .
$$

Example 1. Consider the following singularly perturbed delay problem [11].

$$
\begin{cases}u_{t}-\varepsilon u_{x x}+\frac{1+x^{2}}{2} u(x, t)=-u(x, t-1)+t^{3}, & (x, t) \in(0,1) \times(0,2], \\ u(x, t)=0, & (x, t) \in[0,1] \times[-1,0], \\ u(0, t)-\sqrt{\varepsilon} u_{x}(0, t)=-(128 / 35) \pi^{-1 / 2} t^{7 / 2}, & t \in[0,2], \\ u(1, t)+\sqrt{\varepsilon} u_{x}(1, t)=-(128 / 35) \pi^{-1 / 2} t^{7 / 2}, & t \in[0,2] .\end{cases}
$$

The computed maximum point-wise errors $E_{\varepsilon}^{N, \Delta t}$ are given in Table 2. From this result, it is clear that the proposed method gives an $\varepsilon$-uniform convergence.

Computational results in Table 3 confirm that the present method has improved the finite difference method in the literature.

Table 4 displays the comparison of computational results using classical cubic B-spline method for $\lambda=0$ and extended
B-spline method for $\lambda=0.99$. Both the particular selections of the extension parameter give numerical results, but the later value of $\lambda$ gives better result than the former one as well as improved results of finite difference method in the literature. It is clear from Table 4 that extended B-spline performs good result than classical cubic B-spline.

Figure 1 depicts the numerical simulation of solution profile at $N=2^{7}, \Delta t=0.1 / 2^{4}, \varepsilon=2^{-12}$, and $\lambda=-0.55$, which indicates parabolic boundary layers at $x=0$ and $x=1$.

The maximum point-wise error values are plotted using log-log scale as can be seen in Figure 2.

To compare the numerical simulation for both classical $\mathrm{B}$-spline and extended $\mathrm{B}$-spline, solution profile is plotted at $N=2^{7}, \Delta t=0.1 / 2^{4}, \varepsilon=2^{-12}$, and $\lambda=0$ in Figure 3 , which shows the solution profile of classical B-spline.

Figure 4 is the numerical simulation using line graph at $N=2^{4}, \Delta t=0.1 / 2, \varepsilon=2^{-12}$, and $\lambda=-0.55$, which indicates parabolic-type boundary layers at $x=0$ and $x=1$.

\section{Conclusion}

This article proposed an implicit Euler method for time derivative with uniform mesh and extended cubic B-spline collocation method for space derivative on Shishkin mesh to solve singularly perturbed delay parabolic reactiondiffusion problem subject to mixed boundary conditions. The proposed method is shown to be accurate of $O(\Delta t+$ $N^{-2} \ln ^{2} N$ ) by preserving an $\varepsilon$-uniform convergence. The suitable choice of the extension parameter $\lambda$ minimizes the error. To see the effect of delay and extension parameter $\lambda$ in the boundary regions, graphs are plotted on the solution profile for $\lambda=-0.55 \in[-8,1]$.

\section{Data Availability}

No data were used to support the findings of this study.

\section{Conflicts of Interest}

The authors declare that there is no conflict of interest regarding the publication of this manuscript.

\section{Acknowledgments}

This work was partially supported by Jimma University, College of Natural Sciences, Ethiopia.

\section{References}

[1] B. Zhang and Y. Zhou, Qualitative Analysis of Delay Partial Difference Equations, Hindawi Publishing Corporation, London, 2007.

[2] M. Wazewska-Czyzewska and A. Lasota, "Mathematical models of the red cell system," Mathematica Applicanda, vol. 6, pp. 25-40, 1976.

[3] A. R. Ansari, S. A. Bakr, and G. I. Shishkin, "A parameterrobust finite difference method for singularly perturbed delay parabolic partial differential equations," Journal of Computational and Applied Mathematics, vol. 205, no. 1, pp. 552-566, 2007. 
[4] J. Singh, S. Kumar, and M. Kumar, "A domain decomposition method for solving singularly perturbed parabolic reactiondiffusion problems with time delay," Numerical Methods for Partial Differential Equations, vol. 34, no. 5, pp. 1849-1866, 2018.

[5] S. Gowrisankar and S. Natesan, "Robust numerical scheme for singularly perturbed delay parabolic initial-boundary-value problems on equidistributed grids," Electronic Transactions on Numerical Analysis, vol. 41, pp. 376-395, 2014.

[6] S. Kumar and M. Kumar, "High order parameter-uniform discretization for singularly perturbed parabolic partial differential equations with time delay," Computers and Mathematics with Applications, vol. 68, no. 10, pp. 1355-1367, 2014.

[7] L. Govindarao and J. Mohapatra, "Richardson extrapolation technique for singularly perturbed delay parabolic partial differential equation," International Journal of Advanced Trends in Computer Applications, no. 1, pp. 44-48, 2019.

[8] L. Govindarao, J. Mohapatra, and A. Das, "A fourth-order numerical scheme for singularly perturbed delay parabolic problem arising in population dynamics," Journal of Applied Mathematics and Computing, vol. 63, no. 1-2, pp. 171-195, 2020.

[9] S. Kumar and M. Kumar, "A robust numerical method for a two-parameter singularly perturbed time delay parabolic problem," Computational and Applied Mathematics, vol. 39, no. 209, pp. 1-25, 2020.

[10] V. Gupta, M. Kumar, and S. Kumar, "Higher order numerical approximation for time dependent singularly perturbed differential-difference convection-diffusion equations," Numerical Methods for Partial Differential Equations, vol. 34, no. 1, pp. 357-380, 2018.

[11] P. Avudai Selvi and N. Ramanujam, "A parameter uniform difference scheme for singularly perturbed parabolic delay differential equation with Robin type boundary condition," Applied Mathematics and Computation, vol. 296, no. C, pp. 101-115, 2017.

[12] X. L. Han and S. J. Liu, "An extension of the cubic uniform Bspline curves," Journal of Computer Aided Design and Computer Graphics, vol. 15, no. 5, pp. 576-578, 2003.

[13] X. Gang and W. Guo-Zhao, "Extended cubic uniform B-spline and $\alpha$-B-spline," Acta Automatica Sinica, vol. 34, no. 8, pp. 980-984, 2008.

[14] S. T. Mohyud-Din, T. Akram, M. Abbas, A. Izani Ismail, and N. H. M. Ali, "A fully implicit finite difference scheme based on extended cubic B-splines for time fractional advectiondiffusion equation," Advances in Difference Equations, vol. 2018, no. 109, pp. 1-17, 2018.

[15] S. Sharifi and J. Rashidinia, "Numerical solution of hyperbolic telegraph equation by cubic B-spline collocation method," Applied Mathematics and Computation, vol. 281, no. C, pp. 28-38, 2016.

[16] S. Sharifi and J. Rashidinia, "Collocation method for convection-reaction-diffusion equation," Journal of King Saud University-Science, vol. 31, no. 4, pp. 1115-1121, 2019.

[17] S. C. S. Rao, S. Kumar, and M. Kumar, "A parameter-uniform B-spline collocation method for singularly perturbed semilinear reaction-diffusion problems," Journal of Optimization Theory and Applications, vol. 146, no. 3, pp. 795-809, 2010.

[18] S. C. S. Rao and S. Kumar, "A robust numerical method for singularly perturbed semilinear convection-diffusion problems," Neural, Parallel, and Scientific Computations, vol. 18, no. 2, pp. 155-166, 2010.
[19] D. Kumar and P. Kumari, "A parameter-uniform collocation scheme for singularly perturbed delay problems with integral boundary condition," Journal of Applied Mathematics and Computing, vol. 63, no. 1-2, pp. 813-828, 2020.

[20] D. Kumar and M. K. Kadalbajoo, "A parameter-uniform numerical method for time-dependent singularly perturbed differential-difference equations," Applied Mathematical Modelling, vol. 35, no. 6, pp. 2805-2819, 2011.

[21] D. Kumar and P. Kumari, "A parameter-uniform scheme for singularly perturbed partial differential equations with a time lag," Numerical Methods for Partial Differential Equations, vol. 36, no. 4, pp. 368-386, 2020.

[22] I. T. Daba and G. F. Duressa, "Extended cubic B-spline collocation method for singularly perturbed parabolic differentialdifference equation arising in computational neuroscience," International Journal of Numerical Method in Biomedical Engineering, vol. 37, article e3418, 2021.

[23] T. B. Mekonnen and G. F. Duressa, "Computational method for singularly perturbed two-parameter parabolic convectiondiffusion problems," Cogent Mathematics and Statistics, vol. 7, no. 1, pp. 1-16, 2020.

[24] T. B. Mekonnen and G. F. Duressa, "Uniformly convergent numerical method for two-parametric singularly perturbed parabolic convection-diffusion problems," Journal of Applied and Computational Mechanics, vol. 7, no. 2, pp. 535-545, 2021.

[25] T. A. Bullo, G. F. Duressa, and G. A. Delga, "Accelerated fitted operator finite difference method for singularly perturbed parabolic reaction-diffusion problems," Computational Methods for Differential Equations, 2021.

[26] T. A. Bullo, G. F. Duressa, and G. A. Delga, "Robust finite difference method for singularly perturbed two-parameter parabolic convection-diffusion problems," International Journal of Computational Methods, vol. 18, article 2050034, 2020.

[27] O. A. Ladyzhenskaia, V. A. Solonnikov, and N. N. Ural'tseva, "Linear and quasi-linear equations of parabolic type," American Mathematical Society, vol. 23, 1988.

[28] C. Clavero, J. C. Jorge, and F. Lisbona, "A uniformly convergent scheme on a non-uniform mesh for convectiondiffusion parabolic problems," Journal of Computational and Applied Mathematics, vol. 154, no. 2, pp. 415-429, 2003.

[29] H. S. Shukla and M. Tamsir, "Extended modified cubic Bspline algorithm for nonlinear Fishers reaction-diffusion equation," Alexandria Engineering Journal, vol. 55, no. 3, pp. 28712879, 2016

[30] C. A. Hall, "On error bounds for spline interpolation," Journal of Approximation Theory, vol. 1, no. 2, pp. 209-218, 1968.

[31] J. M. Varah, "A lower bound for the smallest singular value of a matrix," Linear Algebra and its Application, vol. 11, no. 1, pp. 3-5, 1975. 